\title{
Editorial: Applications of Monte Carlo Method in Chemical, Biochemical and Environmental Engineering
}

\author{
Gurkan $\operatorname{Sin}^{1 *}$ and Antonio Espuña ${ }^{2}$ \\ 1 Department of Chemical and Biochemical Engineering, PROSYS Research Center, Technical University of Denmark, Lyngby, \\ Denmark, ${ }^{2}$ Center for Process and Environmental Engineering, Polytechnic University of Catalonia, Barcelona, Spain
}

Keywords: Monte Carlo simulation, global sensitivity analysis (GSA), uncertainty analysis (UA), model identification and estimation, wastewater treatment, fermentation process, renewable energy, model-based engineering (MBE)

\section{Editorial on the Research Topic}

Applications of Monte Carlo Method in Chemical, Biochemical and Environmental Engineering

Monte Carlo methods are defined broadly as a statistical approach to provide approximate solutions to mathematically complex optimization or simulation problems by using random sequences of numbers. The two main advantages of Monte Carlo methods are perhaps that the concept is relatively simple and easy to use, and the same method has a sound basis. The Law of large numbers ensures that the Monte Carlo solution converges asymptotically to the true solution of a problem and since its first formulation by Metropolis and Ulam (1949), Monte Carlo methods have seen widespread application across many scientific and engineering disciplines.

In this Research Topic, we have brought together seven original research articles about applications of Monte Carlo methods within the domain of bio/chemical, energy, and

\section{OPEN ACCESS}

Edited and reviewed by:

Ellen B. Stechel,

Arizona State University,

United States

*Correspondence:

Gurkan Sin

gsi@kt.dtu.dk

Specialty section: This article was submitted to Process and Energy Systems Engineering,

a section of the journal Frontiers in Energy Research

Received: 11 March 2020 Accepted: 09 April 2020 Published: 20 May 2020

Citation:

Sin G and Espuña A (2020) Editorial: Applications of Monte Carlo Method in Chemical, Biochemical and Environmental Engineering.

Front. Energy Res. 8:68. doi: 10.3389/fenrg.2020.00068 environmental systems engineering. Each of these contributions highlights different aspects of the Monte Carlo methods, from model identification to system and process analysis. As regards model identification, we have three contributions from different domain applications. In particular, Odriozola et al. aptly demonstrate the application of the Monte Carlo methods for comparative assessment of six different model candidates to describe inhibition mechanisms for a particular anaerobic wastewater treatment process. Continuing on the model identification problem, Krausch et al. present a methodological contribution, which combines Monte Carlo simulations for better quantifying parameter uncertainties within the context of optimal design of experiment for bioprocess application. On the other hand, Laínez-Aguirre and Puigjaner studied the model candidate generation problem for pharmacokinetic field and its subsequent evaluation using an optimization framework coupled with a Bayesian metric (that is computed using Markov chain Monte Carlo sampling). These contributions nicely demonstrate the versatile use of the Monte Carlo methods to study and understand how different sources of uncertainty, from measurements/data to model structure (model candidates), propagate to model predictions. In this way, these methods help quantify the model output uncertainties, which are then used as an additional metric in model development/selection studies as well as to expand the basis for designing optimal experiments for data collection.

The remaining contributions in this Research Topic focus on process and system level studies through global sensitivity analysis-an important application area for Monte Carlo methods. In this regards, Nguyen Quang et al. studied a metabolic model to describe biological phosphorus removal-a technology used in wastewater treatment engineering to remove phosphorus-based pollution from receiving water bodies, to gain deeper insight into the complex biological process and its underlying mechanisms. Bergamini et al. developed a very interesting application of the global sensitivity analysis technique to help smart data collection from full scale chemical/process 
plants to perform heat integration studies, which is an important tool in analyzing and improving energy efficiency in the process industry. The last two contributions deal with sustainability problems in the transport and energy sectors. Kadyk et al. presented a compelling application of the Monte Carlo methods for the design and evaluation of fuel cell systems for aviation. In particular, the authors use the Monte Carlo sampling to generate representative mission profiles as input data for the study and, through a sensitivity analysis of the associated performance parameters, suggest future directions for technological developments. Last but not least, Lo Piaono et al. demonstrated a very useful application of variance based global sensitivity analysis to study competing long term (up to 2050) supply and demand scenarios for silver availability and its impact for the large scale deployment of solar photovoltaics (PV) for renewable electricity production.

All these seven contributions add to the increasing body of multidisciplinary literature works (see e.g., Frutiger et al., 2016, 2017; Anane et al., 2019, just to name few), that successfully demonstrate the broad spectrum of engineering domains and problems that are successfully addressed by the appropriate choice of the Monte Carlo methods. Indeed, we see that modeling are increasingly accepted and established as a research approach on its own right in many scientific and engineering disciplines. As such, modeling forms a complementary part to the already established mature research methodologies including theoretical and experimental approaches. Therefore, looking ahead, we expect that the use of the Monte Carlo methods will be used regularly as part of model-based research projects. Here we emphasize that the concept of Monte Carlo simulations is what is valuable, which is opposed to existing paradigm in which models are mostly used for producing a single simulation/prediction with a nominal set of input values (parameters, inputs, initial/boundary conditions, etc.). Within this context, when used properly, the Monte Carlo concept expand the analysis horizon and invite the modeler to reflect on model output behavior under a wider set of input domain values. This new paradigm of thinking and using models within Monte Carlo concept (one

\section{REFERENCES}

Anane, E., Barz, T., Sin, G., Gernaey, K. V., Neubauer, P., and Bournazou, M. N. C. (2019). Output uncertainty of dynamic growth models: effect of uncertain parameter estimates on model reliability. Biochem. Eng. J. 150:107247. doi: 10.1016/j.bej.2019.107247

Frutiger, J., Bell, I., O’Connell, J. P., Kroenlein, K., Abildskov, J., and Sin, G. (2017). Uncertainty assessment of equations of state with application to an organic Rankine cycle. Mol. Phys. 115, 1225-1244. doi: 10.1080/00268976.2016.1275856

Frutiger, J., Marcarie, C., Abildskov, J., and Sin, G. (2016). A comprehensive methodology for development, parameter estimation, and uncertainty analysis of group contribution based property models an application to the heat of combustion. J. Chem. Eng. Data 61, 602-613. doi: 10.1021/acs.jced.5b00750

Metropolis, N., and Ulam, S. (1949). The monte carlo method. J. Am. Stat. Assoc. 44, 335-341. doi: 10.1080/01621459.1949.10483310

Saltelli, A., Aleksankina, K., Becker, W., Fennell, P., Ferretti, F., Holst, N., et al. (2019). Why so many published sensitivity analyses are false: a systematic does Monte Carlo simulations instead of single point simulation in the input domain) offer a quality assurance and responsible use of models, which we think we will continue to add value especially for making model-based inferences and supporting engineering decisions.

Current challenges associated with the Monte Carlo methods are 2-folds; some of them have to do with the associated computational cost. This is a technical constraint in nature that can be successfully tackled with appropriate strategies, such as the use of metamodels or surrogate models by statistical machine learning algorithms to replace original models, efficient sampling strategies and algorithms (e.g., Quasi-Monte Carlo methods, space-filling strategies, etc.), and/or the application of parallel computing among others. On other hand, defining a proper context and formulating an appropriate problem for Monte Carlo analysis remains an important challenge especially for the quality of Monte Carlo studies in engineering applications. This topic has recently been addressed by Saltelli et al. (2019) in a comprehensive review analysis, as well as in earlier contribution (Sin et al., 2009) from a process design application perspective. In this regard, proper identification of sources of uncertainties and their characterization for the input domain definition is a critical step in any Monte Carlo simulations, be it used for sensitivity or uncertainty analysis. Many of these Monte Carlo simulations demonstrated that we can deal with known knowns and known unknowns but not with unknown unknowns. Further research into this direction will be highly valuable to generate recommendations and best practices when defining the input uncertainty for a given application domain or engineering problem. In this regard, one should refrain from generalizing the results from Monte Carlo analysis, and instead frame the interpretation of a Monte Carlo analysis within the specific context and problem statement where they are used.

\section{AUTHOR CONTRIBUTIONS}

All authors listed have made a substantial, direct and intellectual contribution to the work, and approved it for publication. review of sensitivity analysis practices. Environ. Model. Softw. 114, 29-39. doi: 10.1016/j.envsoft.2019.01.012

Sin, G., Gernaey, K. V., Neumann, M. B., van Loosdrecht, M. C., and Gujer, W. (2009). Uncertainty analysis in WWTP model applications: a critical discussion using an example from design. Water Res. 43, 2894-2906. doi: 10.1016/j.watres.2009.03.048

Conflict of Interest: The authors declare that the research was conducted in the absence of any commercial or financial relationships that could be construed as a potential conflict of interest.

Copyright (c) 2020 Sin and Espuña. This is an open-access article distributed under the terms of the Creative Commons Attribution License (CC BY). The use, distribution or reproduction in other forums is permitted, provided the original author(s) and the copyright owner(s) are credited and that the original publication in this journal is cited, in accordance with accepted academic practice. No use, distribution or reproduction is permitted which does not comply with these terms. 